\title{
Upper and Lower Bounds for the Center of Flexure
}

\author{
Lawrence E. Payne
}

(February 1, 1960)

\begin{abstract}
There is disagreement in the literature as to how the center of flexure of an isotropic elastic beam should be defined. In either of the two most widely accepted definitions, upper and lower bounds for the coordinates of the center of flexure are obtained.
\end{abstract}

\section{Introduction}

We consider a cantilever beam of uniform cross section and choose the z-axis to coincide with the line of cross-section centroids. The plane end $z=0$ is assumed fixed and the other end $z=l$ is loaded by some distribution of forces which is statically equivalent to a single force with components $\left(W_{x}, W_{y}, 0\right)$ acting at a load point $\left(x_{L}, y_{L}, l\right)$. In the treatment of the flexure problem [9] ${ }^{1}$ (a similar treatment appears in [3]) we seek a load point $\left(x_{f}, y_{f}, l\right)$ which has the property that any load $\left(W_{x}, W_{y}, 0\right)$ applied through this point produces no local twist at the centroid of the section, i.e., the mean value of the local twist over the cross section vanishes (see [9] p. 200). This point $\left(x_{f}, y_{f}, l\right)$ is referred to as the center of flexure [9]. The determination of $\left(x_{f}, y_{f}, l\right)$ permits us to break up the general flexure problem into two separate problemsone of pure torsion and one of pure flexure. This point is sometimes referred to as the center of shear (see, for instance, [3]), and it is well known that there is difference of opinion in the literature as to the way in which the point should be defined (see, for instance, $[1,2,4,10,11]$. A discussion of these differences has been given in a recent paper by Pearson [7], who demonstrates that in general the two expressions for the center of shear yield results which are very near to one another.

It is not our point here to enter into the discussion of the relative merits of the two definitions. We merely point out that the expression of Trefftz [10] is simpler and independent of Poisson's ratio. The center of flexure of [9], on the other hand, permits an easy decomposition of the general flexure problem.

In order to avoid confusion we shall hereafter refer to the point defined in [9] as the center of flexure and that given by Trefftz [10] as the center of shear.

In this paper then we derive upper and lower bounds for the coordinates of the center of flexure $\left(x_{f}, y_{f}, l\right)$ and the center of shear $\left(x_{s}, y_{s}, l\right)$. In the first case we use the formulation of the flexure problem derived in [9] and thus concern ourselves with the problem of pure flexure.

It is of course well known (see, for instance, [7]) that if we have the solution to the pure torsion problem for the beam in question then we can determine both the center of shear and the center of flexure without knowledge of the solution to the flexure problem. As would be expected it is possible in each case to obtain upper and lower bounds for the coordinates of the point by approximating the solution to the torsion problem alone.

\section{Bounds for the Center of Flexure}

We denote by $\mathscr{D}$ the cross section of the beam and let $C$ be its boundary. (The region may be simply or multiply connected.) The origin of a rectangular coordinate system is taken at the centroid of $\mathscr{D}$. For simplicity the $x$ and $y$ axes are chosen to be principal axes of inertia, i.e.,

$$
\iint_{\mathscr{D}} x y d A=0 .
$$

\footnotetext{
1 Figures in brackets indicate the literature references at the end of this paper.
} 
According to [9] the pure flexure problem is solved once the solutions to the following boundary value problems have been determined:

$$
\begin{gathered}
\frac{\partial^{2} \varphi_{1}}{\partial x^{2}}+\frac{\partial^{2} \varphi_{1}}{\partial y^{2}}=0, \quad \frac{\partial^{2} \varphi_{2}}{\partial x^{2}}+\frac{\partial^{2} \varphi_{2}}{\partial y^{2}}=0 \quad \text { in } \mathscr{D} \\
\frac{\partial \varphi_{1}}{\partial n}=\left[(1+\sigma) x^{2}-\sigma y^{2}\right] n_{x} \\
\frac{\partial \varphi_{2}}{\partial n}=\left[(1+\sigma) y^{2}-\sigma x^{2}\right] n_{y} \quad \text { on } C
\end{gathered}
$$

where $\sigma$ is Poisson's ratio and $n_{x}$ and $n_{y}$ are the $x$ and $y$ components of the unit normal on $C$. (The normal is assumed directed outward from $\mathscr{D}$.) The coordinates of the center of flexure $\left(x_{f}, y_{f}\right)$ are defined by:

$$
\begin{aligned}
& x_{f}=\frac{1}{2(1+\sigma) I_{x}} \iint_{\mathscr{D}}\left[x \frac{\partial \varphi_{2}}{\partial y}-y \frac{\partial \varphi_{2}}{\partial x}-(1+\sigma) x y^{2}+\sigma x^{3}\right] d A, \\
& y_{f}=\frac{1}{2(1+\sigma) I_{y}} \iint_{\mathscr{D}}\left[x \frac{\partial \varphi_{1}}{\partial y}-y \frac{\partial \varphi_{1}}{\partial x}+(1+\sigma) x^{2} y-\sigma y^{3}\right] d A
\end{aligned}
$$

where

$$
\begin{aligned}
I_{x} & =\iint_{\mathscr{D}} y^{2} d A \\
I_{y} & =\iint_{\mathscr{D}} x^{2} d A .
\end{aligned}
$$

Thus

$$
2(1+\sigma) I_{x} x_{f}=\iint_{\mathscr{D}}\left[\frac{\partial}{\partial y}\left(x \varphi_{2}\right)-\frac{\partial}{\partial x}\left(y \varphi_{2}\right)-(1+\sigma) x y^{2}+\sigma x^{3}\right] d A .
$$

Using the divergence theorem we have

$$
2(1+\sigma) I_{x} x_{f}=\oint_{C} \varphi_{2}\left(x n_{y}-y n_{x}\right) d s-J
$$

where

$$
J=\iint_{\mathscr{D}}\left[(1+\sigma) x y^{2}-\sigma x^{3}\right] d A .
$$

Since $J$ is a geometric quantity it can be computed explicitly.

We note now that the coefficient of $\varphi_{2}$ in the boundary integral in (2.9) is proportional to the boundary value of the normal derivative of the warping function $\varphi$ in the pure torsion problem. In fact

$$
\frac{\partial \varphi}{\partial n}=y n_{x}-x n_{y} \quad \text { on } C \text {. }
$$

Thus if we let

$$
\chi=2(1+\sigma) I_{x} x_{f}+J
$$

we find

$$
\chi=-\oint_{C} \frac{\partial \varphi}{\partial n} d s=-D\left(\varphi, \varphi_{2}\right)
$$

where

$$
D\left(\varphi, \varphi_{2}\right)=\iint_{\mathscr{D}}\left[\frac{\partial \varphi}{\partial x} \frac{\partial \varphi_{2}}{\partial x}+\frac{\partial \varphi}{\partial y} \frac{\partial \varphi_{2}}{\partial y}\right] d A .
$$

The determination of bounds for $x_{f}$ is thus equivalent to the determination of bounds for $\chi$. 
Note that if the warping function $\varphi$ is known the value of $x_{f}$ can be determined explicitly since

$$
\chi=-\oint_{C} \varphi\left[(1+\sigma) y^{2}-\sigma x^{2}\right] n_{y} d s .
$$

If $\varphi$ is not known we introduce two arbitrary harmonic functions $u$ and $u_{2}$ and define

$$
\begin{aligned}
& a_{1}=-D\left(\varphi, u_{2}\right)=-\oint_{C} u_{2}\left(y n_{x}-x n_{y}\right) d s, \\
& a_{2}=-D\left(u, \varphi_{2}\right)=-\oint_{C} u\left[(1+\sigma) y^{2}-\sigma x^{2}\right] n_{y} d s, \\
& a_{3}=-D\left(u, u_{2}\right) .
\end{aligned}
$$

With $u$ and $u_{2}$ prescribed we can determine $a_{1}, a_{2}$, and $a_{3}$ explicitly. We now form the expression

$$
\begin{aligned}
\chi-a_{1}-a_{2}+a_{3} & =-D\left(\varphi-u, \varphi_{2}-u_{2}\right) \\
& =-\oint_{C}(\varphi-u)\left\{\left[(1+\sigma) y^{2}-\sigma x^{2}\right] n_{y}-\frac{\partial u_{2}}{\partial n}\right\} d s .
\end{aligned}
$$

An application of Schwarz's inequality yields

$$
\left(\chi-a_{1}-a_{2}+a_{3}\right)^{2} \leq \oint_{C}(\varphi-u)^{2} d s \oint_{C}\left\{\left[(1+\sigma) y^{2}-\sigma x^{2}\right] n_{y}-\frac{\partial u_{2}}{\partial n}\right\}^{2} d s .
$$

It remains now to approximate the first boundary integral on the right of $(2.20)$ in terms of known quantities.

By adding an appropriate constant (if necessary) it is possible to choose $\varphi-u$ in such a a way that

$$
\oint_{C}(\varphi-u) d s=0 .
$$

With $\varphi-u$ so chosen we shall estimate the first integral on the right of (2.20) in terms of the boundary integral of the square of its normal derivative. To this end we note that

$$
\frac{\oint_{C}\left(\frac{\partial}{\partial s}(\varphi-u)\right)^{2} d s}{\oint_{C}(\varphi-u)^{2} d s} \geq \min _{\oint_{c} d s=0} \frac{\oint_{C}\left(\frac{\partial v}{\partial s}\right)^{2} d s}{\oint_{C} v^{2} d s}=\frac{4 \pi^{2}}{L^{2}}
$$

where $L$ denotes the length of the perimeter of $C, \partial / \partial s$ denotes the tangential derivative on $C$, and $v$ is any function continuous on $C$ and satisfying the condition $\oint_{C} v d s=0$. The quantity on the right of (2.22) is just the eigenvalue of the vibrating string equation, the differential equation which arises as the Euler equation for the minimum of the quotient $\left(\oint_{C}(\partial v / \partial s)^{2} d s\right)$ / $\oint_{C} v^{2} d s$. It follows then that

$$
\oint_{C}(\varphi-u)^{2} d s \leq \frac{L^{2}}{4 \pi^{2}} \oint_{C}\left[\frac{\partial}{\partial s}(\varphi-u)\right]^{2} d s .
$$

We seek now an inequality which relates the integral on the right-hand side of $(2.23)$ to the boundary integral of the square of the normal derivative of $\varphi-u$. We introduce therefore, 
an arbitrary continuous vector function with components $f_{k}$ and consider the generalized Rellich identity (see [6, 8]). Letting $w=\varphi-u$, we have

$0=\int_{\mathscr{D}} \int_{k} \frac{\partial w}{\partial x_{k}} \Delta w d A=\oint_{C} f_{k} \frac{\partial w}{\partial x_{k}} \frac{\partial w}{\partial n} d s-\frac{1}{2} \oint_{C} f_{k} n_{k}|\operatorname{grad} w|^{2} d s-\iint_{\mathscr{D}} \frac{\partial f_{k}}{\partial x_{l}} \frac{\partial w}{\partial x_{k}} \frac{\partial w}{\partial x_{l}} d A+\frac{1}{2} \iint_{\mathscr{D}} \frac{\partial f_{k}}{\partial x_{k}}|\operatorname{grad} w|^{2} d A$

where $\Delta$ denotes the Laplace operator $\left(\Delta=\left(\partial^{2} / \partial x^{2}\right)+\left(\partial^{2} / \partial y^{2}\right)\right)$ and summation is to be carried out over the repeated indices $k, l=1,2$. The $f_{k}$ are now assumed to be so chosen that

$$
p=f_{k} n_{k}>0 \text { on } C \text {. }
$$

A decomposition of the derivatives of $u$ in the boundary integrals into normal and tangential components yields (see [8])

$$
\oint_{C} p\left(\frac{\partial w}{\partial s}\right)^{2} d s=\oint_{C} p\left(\frac{\partial w}{\partial n}\right)^{2} d s+2 \oint_{C} f_{k} \frac{\partial x_{k}}{\partial s} \frac{\partial w}{\partial s} \frac{\partial w}{\partial n} d s+\left.\int_{\mathscr{D}} \int_{\partial f_{k}} \frac{\partial f_{k}}{\partial x_{k}} \operatorname{grad} w\right|^{2} d A-2 \int_{\mathscr{D}} \frac{\partial f_{k}}{\partial x_{l}} \frac{\partial w}{\partial x_{k}} \frac{\partial w}{\partial x_{l}} d A .
$$

Since the functions $f_{k}$ are prescribed, we can find a constant $\gamma$ such that (see $[6$, p. 555])

$$
\iint_{\mathscr{D}} \frac{\partial f_{k}}{\partial x_{k}}|\operatorname{grad} w|^{2} d A-2 \iint_{\mathscr{D}} \frac{\partial f_{k}}{\partial x_{l}} \frac{\partial w}{\partial x_{k}} \frac{\partial w}{\partial x_{l}} d a \leq \gamma D(w, w) \text {. }
$$

We make use also of the ordinary Green's identity

$$
D(w, w)=\oint_{C} w \frac{\partial w}{\partial n} d s
$$

which yields by Schwarz's inequality

$$
D(w, w) \leq\left(\oint_{C} w^{2} d s\right)^{\frac{1}{2}}\left(\oint_{C}\left(\frac{\partial w}{\partial n}\right)^{2} d s\right)^{\frac{1}{2}} .
$$

In view of (2.23), eq (2.29) yields

$$
D(w, w) \leq \frac{L}{2 \pi}\left[\oint_{C}\left(\frac{\partial w}{\partial s}\right)^{2} d s\right]^{\frac{1}{2}}\left[\oint_{C}\left(\frac{\partial w}{\partial n}\right)^{2} d s\right]^{\frac{1}{2}} .
$$

We now insert (2.27) and (2.30) back into (2.26), make use of the Schwarz inequality and obtain

$$
\begin{aligned}
\oint_{C} p\left(\frac{\partial w}{\partial s}\right)^{2} d s \leq \oint_{C} p\left(\frac{\partial w}{\partial n}\right)^{2} d s+2\left[\oint_{C} \frac{\left(f^{k} \frac{\partial x_{k}}{\partial s}\right)^{2}}{p}\left(\frac{\partial w}{\partial n}\right)^{2} d s\right]^{\frac{1}{2}}\left[\oint_{C} p\left(\frac{\partial w}{\partial s}\right)^{2} d s\right]^{\frac{1}{2}} & \\
& +\frac{\gamma L}{2 \pi p_{\min }^{\frac{1}{2}}}\left[\oint_{C} p\left(\frac{\partial w}{\partial s}\right)^{2} d s\right]^{\frac{1}{2}}\left[\oint_{C}\left(\frac{\partial w}{\partial n}\right)^{2} d s\right]^{\frac{1}{2}},
\end{aligned}
$$

where $p_{\min }$ is the minimum value of $p$ on $C$. This inequality yields

$$
\begin{aligned}
& {\left[\oint_{C} p\left(\frac{\partial w}{\partial s}\right)^{2} d s\right]^{\frac{1}{2}} \leq\left[\oint_{C} \frac{\left(f^{k} \frac{\partial x_{k}}{\partial s}\right)^{2}}{p}\left(\frac{\partial w}{\partial n}\right)^{2} d s\right]^{\frac{1}{2}}+\left[\frac{\gamma^{2} L^{2}}{4 \pi^{2} p_{\min _{C}}} \oint_{C}\left(\frac{\partial w}{\partial n}\right)^{2} d s\right]^{\frac{1}{2}}+\left\{\left(\left[\oint_{C} \frac{\left(f^{k} \frac{\partial x_{k}}{\partial s}\right)^{2}}{p}\left(\frac{\partial w}{\partial n}\right)^{2} d s\right]^{\frac{1}{2}}\right.\right.} \\
& \left.\left.+\left[\frac{\gamma^{2} L^{2}}{4 \pi^{2} p_{\min }} \oint_{C}\left(\frac{\partial w}{\partial n}\right)^{2} d s\right]^{\frac{1}{2}}\right)^{2}+\oint_{C} p\left(\frac{\partial w}{\partial n}\right)^{2} d s\right\}^{\frac{1}{2}} .
\end{aligned}
$$


From (2.32) it is thus always possible to obtain the inequality

$$
\oint_{C}\left(\frac{\partial w}{\partial s}\right)^{2} d s \leq \alpha \oint_{C}\left(\frac{\partial w}{\partial n}\right)^{2} d s
$$

where

$$
\alpha p_{\min }=\left(\beta+\left[\beta^{2}+p_{\max }\right]^{\frac{1}{2}}\right)^{2}
$$

and

$$
\beta=\left\{\frac{\left|f^{k} \frac{\partial x_{k}}{\partial s}\right|}{p^{\frac{1}{2}}}\right\}+\frac{\gamma L}{2 \pi p_{\min }^{\frac{1}{2}}} .
$$

If the boundary $C$ is star-shaped with respect to some point $\left(x_{o}, y_{0}\right)$ in $\mathscr{D}$, the problem is simplified considerably. The fact that the boundary is star-shaped with respect to $\left(x_{0}, y_{0}\right)$ is equivalent to the condition that

$$
q=\left(x-x_{0}\right) n_{x}+\left(y-y_{0}\right) n_{y}>0
$$

at every point on $C$. Thus, in this case, (2.25) is satisfied if we let

$$
f_{1}=x-x_{0}, \quad f_{2}=y-y_{0} .
$$

The left-hand side of (2.27) then vanishes identically, and hence we take $\gamma \equiv 0$ in $(2.31),(2.32)$, and (2.35). We find in this case instead of (2.33) (see [5]) that

$$
\oint_{C}\left(\frac{\partial w}{\partial s}\right)^{2} d s \leq q_{\min }^{-1}\left[1+\left|\frac{\partial r}{\partial s}\right|_{\max }\right]_{C}^{2} \oint_{C} \frac{r^{2}}{q}\left(\frac{\partial w}{\partial n}\right)^{2} d s .
$$

In the general case then, an inequality of the form

$$
\oint_{C}\left(\frac{\partial(\varphi-u)}{\partial s}\right)^{2} d s \leq \oint_{C} A\left[\frac{\partial}{\partial n}(\varphi-u)\right]^{2} d s
$$

is obtained, where $A$ is a completely determined positive function. Inserting (2.39) and $(2.23)$ into $(2.20)$, we find

$$
\left(\chi-a_{1}-a_{2}+a_{3}\right)^{2} \leq \frac{\left.L^{2}\right\}}{4 \pi^{2}} \oint_{C} A\left[\frac{\partial u}{\partial n}-y n_{x}+x n_{y}\right]^{2} d s \oint_{C}\left\{\frac{\partial u_{2}}{\partial n}-\left[(1+\sigma) y^{2}-\sigma x^{2}\right] n_{y}\right\}^{2} d s .
$$

(It is apparent that the quantity $A$ may be placed in the second integral rather than in the first if it is desirable.) We now use the Rayleigh-Ritz technique to make the two integrals on the right of (2.40) small.

Note that it is possible to derive close upper and lower bounds for $x_{f}$ without approximating the flexure function at all. In fact, if we use instead of $u$ a conjugate function $v$ defined by ${ }^{2}$

we obtain (setting $u_{2} \equiv 0$ )

$$
\frac{\partial u}{\partial x}=\frac{\partial v}{\partial y}, \quad \frac{\partial u}{\partial y}=-\frac{\partial v}{\partial x}
$$

$$
\left|\chi-a_{2}\right|^{2} \leq \frac{L^{2}}{4 \pi^{2}} \oint_{C}\left[\frac{\partial}{\partial s}\left(v-\frac{1}{2} r^{2}\right)\right]^{2} d s \oint_{C} A\left\{\left[(1-\sigma) y^{2}-\sigma x^{2}\right] n_{y}\right\}^{2} d s .
$$

In this case the $A$ term has been put in the second integral since this expression is a purely geometrical quantity (independent of $v$ ) and can be computed explicitly.

2 The function $v$ is not to be confused with that used in (2.22). 
In an entirely analogous way we find that

$$
-2(1+\sigma) I_{y} y_{f}+\iint_{\mathscr{D}}\left[(1+\sigma) x^{2} y-\sigma y^{3}\right] d A=D\left(\varphi, \varphi_{1}\right) .
$$

By introducing the two arbitrary harmonic functions $u$ and $u_{1}$ and proceeding as before, we obtain upper and lower bounds for $y_{f}$. Note that if we choose $u_{1}, u_{2} \equiv 0$ and merely approximate the warping function (or the conjugate function) on the right, then the same minimizing function $u$ (or $v$ ) minimizes the error term in each case.

\section{Bounds for the Center of Shear}

We look now at the expression for the center of shear $\left(x_{s}, y_{s}, l\right)$ as defined by Trefftz [10]. The coordinates $x_{s}$ and $y_{s}$ are given by

$$
\begin{aligned}
& x_{s}=-\frac{1}{I_{x}} \int_{\mathscr{D}} \varphi y d A \\
& y_{s}=\frac{1}{I_{y}} \iint_{\mathscr{D}} \varphi x d A .
\end{aligned}
$$

Equation (3.2) may be rewritten as

$$
y_{s}=\frac{1}{6 I_{y}} \iint_{\mathscr{D}}\left(\Delta x^{3}\right) \varphi d A .
$$

By Green's formula we have

$$
\begin{aligned}
y_{s} & =\frac{1}{6 I_{y}} \oint_{C}\left[3 x^{2} n_{x} \varphi-x^{3} \frac{\partial \varphi}{\partial n}\right] d s \\
& =\frac{1}{6 I_{y}} \oint_{C}\left[3 x^{2} n_{x} \varphi-x^{3}\left(y n_{x}-x n_{y}\right)\right] d s .
\end{aligned}
$$

The divergence theorem then yields

$$
y_{s}+\frac{1}{2 I_{y}} \iint_{\mathscr{D}} x^{2} y d A=\frac{1}{2 I_{y}} \oint_{C} x^{2} n_{x} \varphi d s .
$$

We introduce the notation

$$
\Phi=y_{s}+\frac{1}{2 I_{y}} \iint_{\mathscr{D}} x^{2} y d A,
$$

and two harmonic functions $h_{1}$ and $h_{2}$. Let

$$
\begin{aligned}
& b_{1}=\oint_{C} h_{1} x^{2} n_{x} d s, \\
& b_{2}=\oint_{C} \varphi \frac{\partial h_{2}}{\partial n} d s=\oint_{C} h_{2}\left[y n_{x}-x n_{y}\right] d s, \\
& b_{3}=\oint_{C} h_{1} \frac{\partial h_{2}}{\partial n} d s .
\end{aligned}
$$


Combining (3.5) to (3.9) we obtain

$$
\Phi-b_{1}-b_{2}+b_{3}=\oint_{C}\left(\varphi-h_{1}\right)\left(x^{2} n_{x}-h_{2}\right) d s .
$$

Then by Schwarz's inequality

$$
\left[\Phi-b_{1}-b_{2}-b_{3}\right]^{2} \leq \oint_{C}\left(\varphi-h_{1}\right)^{2} d s \oint_{C}\left(x^{2} n_{x}-\frac{\partial h_{2}}{\partial n}\right)^{2} d s .
$$

Again it is desirable to choose $\left(\varphi-h_{1}\right)$ in such a way that

$$
\oint_{C}\left(\varphi-h_{1}\right) d s=0 \text {. }
$$

Using (2.23) and (2.39), we obtain finally

$$
\left[\Phi-b_{1}-b_{2}+b_{3}\right]^{2} \leq \frac{L^{2}}{4 \pi^{2}} \oint_{C} A\left(\frac{\partial \varphi}{\partial n}-\frac{\partial h_{1}}{\partial n}\right)^{2} d s \oint_{C}\left(x^{2} n_{x}-\frac{\partial h_{2}}{\partial n}\right)^{2} d s .
$$

We insert the known boundary value for $\varphi$ on the right-hand side and use the Rayleigh-Ritz technique to obtain close upper and lower bounds for $y_{s}$. Note that if the boundary value problem

$$
\begin{aligned}
& \Delta h_{2}=0 \quad \text { in } \quad \mathscr{D} \\
& \frac{\partial h_{2}}{\partial n}=x^{2} n_{x} \quad \text { on } \quad C
\end{aligned}
$$

can be solved, it is then possible to determine $y_{s}$ explicity without solving either the torsion or the flexure problem.

In a similar way it is possible to obtain upper and lower bounds for the coordinate $x_{s}$.

\section{References}

[1] P. Cicala, Atti. accad. sci. Torino 70, pp. 356-371 (1935).

[2] J. N. Goodier, J. Aeronaut. Sci. 11, pp. 272-280 (1944).

[3] A. E. H. Love, A treatise on the mathematical theory of elasticity (Cambridge Univ. Press, London, England, 1927).

[4] W. R. Osgood, J. Appl. Math. [A] 10, pp. 62-64 (1943).

[5] L. E. Payne and H. F. Weinberger, J. Math. Phys. 4, pp. 291-307 (1955).

[6] L. E. Payne and H. F. Weinberger, Pacific J. Math. 8, pp. 551-573 (1958).

[7] C. E. Pearson, Z. angew. Math. u. Mech. 36, pp. 94-96 (1956).

[8] F. Rellich, Math. Z. 46, pp. 635-646 (1940).

[9] I. S. Sokolnikoff, Mathematical theory of elasticity, 2d ed. (McGraw-Hill Book Co., Inc., New York, N.Y., 1956).

[10] E. Trefftz, Z. angew. Math. u. Mech. 15, pp. 220-225 (1935).

[11] A. Weinstein, Quart. Appl. Math. 5, pp. 97-99 (1947).

$$
\text { Washington, D.C. }
$$

(Paper 64B2-28) 